\title{
Pacific
}

Journal of

Mathematics

\section{COMMUTATORS AND INVARIANT DOMAINS FOR SCHRÖDINGER PROPAGATORS}

\author{
Min-Jei HuANG
}




\title{
COMMUTATORS AND INVARIANT DOMAINS FOR SCHRÖDINGER PROPAGATORS
}

\author{
Min-JeI HUANG
}

\begin{abstract}
We present an operator-theoretic approach to the problem of invariant domains for the Schrödinger evolution equation. The results are applied to the Hamiltonian operators with time-dependent potentials and electric fields.
\end{abstract}

\section{Introduction.}

This paper is concerned with the problem of invariant domains for the Schrödinger evolution equation

$$
i \frac{d}{d t} \varphi(t)=H(t) \varphi(t), \quad \varphi(s)=\varphi_{s}
$$

where $H(t), t \in \mathbb{R}$, is a family of self-adjoint operators acting on a Hilbert space $\mathcal{H}$.

It is known that under suitable conditions on $H(t)$ (see e.g. Kato [4], ReedSimon [9] and Yajima [11]), there exists a unique unitary propagator $U(t, s)$ on $\mathcal{H}$, and a dense subspace $\mathcal{D}$ of $\mathcal{H}$ which is invariant under the propagator so that for each $\varphi_{s} \in \mathcal{D}, \varphi(t)=U(t, s) \varphi_{s}$ is strongly differentiable and satisfies (1).

The problem considered here has been studied by many authors; see FarisLavine [1], Fröhlich [2], Hunziker [3], Kuroda-Morita [5], Ozawa [6, 7], Radin-Simon [8] and Wilcox [10]. Most of them dealt with the time-independent case $H(t) \equiv H$ in which the propagator $U(t, s)=\exp [i(s-t) H]$ is given by the usual one-parameter unitary group. In a recent paper [7], Ozawa investigated the space-time behavior of $U(t, s)$ for the Stark Hamiltonian $H(t)=-\Delta+E \cdot x+V(x, t)$ on $L^{2}\left(\mathbb{R}^{n}, d x\right)$. By using perturbation techniques and space-time estimates for the free propagator $\exp [i t(-\Delta+E \cdot x)]$, Ozawa established several results on the invariance property and smoothing effect for $U(t, s)$ in certain weighted Sobolev spaces. For earlier related rèsults in the case $E=0$, see Kuroda-Morita [5].

We denote the domain of an operator $A$ by $\mathcal{D}(A)$, and if $N$ is positive and self-adjoint, we denote its form domain by $\mathcal{Q}(N)$. Given a positive selfadjoint operator $N$, we are interested in conditions on $H(t)$ for $\mathcal{Q}(N)$ or 
$\mathcal{D}\left(N^{k}\right), k=1,2, \ldots$, to be an invariant subspace of $U(t, s)$ for all $t, s \in \mathbb{R}$. We study this problem in a general operator-theoretic setting in Section 2 . Our approach is based on the commutator theorems of Faris and Lavine [1] and Fröhlich [2]. In Section 3, we apply the abstract theorems of Section 2 to Hamiltonians of the form

$$
H(t)=-\Delta+E(t) \cdot x+V(x, t)
$$

with $N=p^{2}+x^{2}$ or $N=p^{2}$, where $p$ is the momentum operator $-i \nabla$. Our results are related to some of those in $[5,7]$.

\section{Abstract Theorems.}

Let $H(t), t \in \mathbb{R}$, be a family of self-adjoint operators acting on a Hilbert space $\mathcal{H}$. Throughout this section, we will assume that $\bigcap_{t} \mathcal{D}(H(t)) \supseteq \mathcal{D}$ for some dense subspace $\mathcal{D}$ of $\mathcal{H}$, and that $H(t)$ generates a unitary propagator $U(t, s)$ so that

$$
i \frac{d}{d t} U(t, s) \varphi=H(t) U(t, s) \varphi \text { for all } \varphi \in \mathcal{D} .
$$

We denote by $\mathcal{B}(\mathcal{H})$ the space of all bounded linear operators on $\mathcal{H}$ with the usual operator norm $\|\cdot\|$. For a positive self-adjoint operator $N$ on $\mathcal{H}$ and $\epsilon>0$, we define $N_{\epsilon}=N(\epsilon N+1)^{-1}$. Note that $N_{\epsilon} \in \mathcal{B}(\mathcal{H})$ is positive and selfadjoint. Concerning the invariance of the form domain $\mathcal{Q}(N)=\mathcal{D}\left(N^{1 / 2}\right)$, we prove:

Theorem 2.1. Let $N$ be a positive self-adjoint operator so that

(i) $\mathcal{D}(N) \subseteq \bigcap_{t} \mathcal{D}(H(t))$.

(ii) $\pm i[H(t), N] \leq c(t) N$ for some $c \in L_{\text {loc }}^{1}(\mathbb{R})$; that is, $\pm i\{\langle H(t) \varphi, N \varphi\rangle-\langle N \varphi, H(t) \varphi\rangle\} \leq c(t)\langle\varphi, N \varphi\rangle$ for all $\varphi \in \mathcal{D}(N)$. Then $U(t, s)[\mathcal{Q}(N)]=\mathcal{Q}(N)$ for all $t, s$.

Proof. Fix $s$ and set $\varphi(t)=U(t, s) \varphi$ for $\varphi \in \mathcal{H}$. Then we have for $\varphi \in \mathcal{D}$

$$
\begin{aligned}
(d / d t)\left\langle\varphi(t), N_{\varepsilon} \varphi(t)\right\rangle & =\left\langle\varphi(t), i\left[H(t), N_{\epsilon}\right] \varphi(t)\right\rangle \\
& =\left\langle(\epsilon N+1)^{-1} \varphi(t), i[H(t), N](\epsilon N+1)^{-1} \varphi(t)\right\rangle .
\end{aligned}
$$

The hypothesis (ii) now gives that

$$
\begin{aligned}
\left|(d / d t)\left\langle\varphi(t), N_{\epsilon} \varphi(t)\right\rangle\right| & \leq c(t)\left\langle(\epsilon N+1)^{-1} \varphi(t), N(\epsilon N+1)^{-1} \varphi(t)\right\rangle \\
& \leq c(t)\left\langle\varphi(t), N_{\epsilon} \varphi(t)\right\rangle .
\end{aligned}
$$

Integrating we obtain

$$
\left\langle\varphi(t), N_{\epsilon} \varphi(t)\right\rangle \leq\left\langle\varphi, N_{\epsilon} \varphi\right\rangle \exp \left|\int_{s}^{t} c(u) d u\right|
$$


Since $\mathcal{D}$ is dense in $\mathcal{H}$ and $N_{\epsilon}$ is bounded, this estimate holds for all $\varphi \in \mathcal{H}$. Now let $\varphi \in \mathcal{Q}(N)$. Taking $\epsilon \rightarrow 0$, we find that $\varphi(t) \in \mathcal{Q}(N)$ with

$$
\left\|N^{1 / 2} \varphi(t)\right\|^{2} \leq\left\|N^{1 / 2} \varphi\right\|^{2} \exp \left|\int_{s}^{t} c(u) d u\right| .
$$

This shows that $\mathcal{Q}(N)$ is invariant under $U(t, s)$. Since $U(t, s) U(s, t)=I$, we conclude that $U(t, s)[\mathcal{Q}(N)]=\mathcal{Q}(N)$.

Now for any positive integer $k$, we define (leaving aside the domain questions)

$$
Z^{k}(t)=N^{k-1}[H(t), N] N^{-k} \text { and } Z_{\epsilon}^{k}(t)=N_{\epsilon}^{k-1}\left[H(t), N_{\epsilon}\right] N_{\epsilon}^{-k} .
$$

In our applications, these operators are defined on certain dense subspaces and extend to bounded operators on $\mathcal{H}$. We also define

$$
(\operatorname{ad} N) H(t)=[N, H(t)] \text { and }(\operatorname{ad} N)^{k} H(t)=\left[N,(\operatorname{ad} N)^{k-1} H(t)\right] .
$$

As a preparation for our next theorem and further applications, we prove the following:

\section{Lemma 2.2.}

(a) $Z_{\epsilon}^{k}(t)=(\epsilon N+1)^{-k} \sum_{j=0}^{k-1}\left(\begin{array}{c}k-1 \\ j\end{array}\right)(\epsilon N)^{j} Z^{k-j}(t)$. In particular, if $Z^{1}(t), \ldots$, $Z^{k}(t) \in \mathcal{B}(\mathcal{H})$, then $Z_{\epsilon}^{k}(t) \in \mathcal{B}(\mathcal{H})$ and $\left\|Z_{\epsilon}^{k}(t)\right\| \leq \sum_{j=0}^{k-1}\left(\begin{array}{c}k-1 \\ j\end{array}\right)\left\|Z^{k-j}(t)\right\|$.

(b) $\left\{(\operatorname{ad} N)^{k} H(t)\right\} N^{-k}=\sum_{j=0}^{k-1}(-1)^{j+1}\left(\begin{array}{c}k-1 \\ j\end{array}\right) Z^{k-j}(t)$.

Proof. Part (a) is obvious for $k=1$. The general case follows by induction on $k$ :

$$
\begin{aligned}
Z_{\epsilon}^{k+1}(t) & =N_{\epsilon} Z_{\epsilon}^{k}(t) N_{\epsilon}^{-1} \\
& =N_{\epsilon}(\epsilon N+1)^{-k} \sum_{j=0}^{k-1}\left(\begin{array}{c}
k-1 \\
j
\end{array}\right)(\epsilon N)^{j} Z^{k-j}(t) N_{\epsilon}^{-1} \\
& =(\epsilon N+1)^{-k-1} \sum_{j=0}^{k-1}\left(\begin{array}{c}
k-1 \\
j
\end{array}\right)(\epsilon N)^{j} N Z^{k-j}(t) N^{-1}(1+\epsilon N) \\
& =(\epsilon N+1)^{-k-1} \sum_{j=0}^{k-1}\left(\begin{array}{c}
k-1 \\
j
\end{array}\right)\left\{(\epsilon N)^{j} Z^{k+1-j}(t)+(\epsilon N)^{j+1} Z^{k-j}(t)\right\} \\
& =(\epsilon N+1)^{-k-1} \sum_{j=0}^{k}\left(\begin{array}{c}
k \\
j
\end{array}\right)(\epsilon N)^{j} Z^{k+1-j}(t)
\end{aligned}
$$


where we have used the identity $\left(\begin{array}{c}k-1 \\ j\end{array}\right)+\left(\begin{array}{c}k-1 \\ j-1\end{array}\right)=\left(\begin{array}{l}k \\ j\end{array}\right)$. The last statement of part (a) follows from the fact that $\left\|(\epsilon N+1)^{-k}(\epsilon N)^{j}\right\| \leq 1$ for $0 \leq j \leq k-1$.

Part (b) can also be proven by an induction argument.

Theorem 2.3. Let $N$ be a positive self-adjoint operator, and define $Z^{j}(t)$ as in $(2)$. Suppose that $Z^{j}(t) \in \mathcal{B}(\mathcal{H})$ with $\left\|Z^{j}(\cdot)\right\| \in L_{\text {loc }}^{1}(\mathbb{R})$ for each $j=$ $1,2, \ldots, k$. Then $U(t, s)\left[\mathcal{D}\left(N^{k}\right)\right]=\mathcal{D}\left(N^{k}\right)$ for all $t, s$.

Proof. As in the proof of Theorem 2.1, set $\varphi(t)=U(t, s) \varphi$ for $\varphi \in \mathcal{H}$. Then we have for $\varphi \in \mathcal{D}$

$$
\begin{aligned}
(d / d t)\left\langle N_{\epsilon}^{k} \varphi(t), N_{\epsilon}^{k} \varphi(t)\right\rangle & =\left\langle\varphi(t), i\left[H(t), N_{\epsilon}^{2 k}\right] \varphi(t)\right\rangle \\
& =i \sum_{j=0}^{2 k-1}\left\langle\varphi(t), N_{\epsilon}^{j}\left[H(t), N_{\epsilon}\right] N_{\epsilon}^{2 k-j-1} \varphi(t)\right\rangle \\
& =2 \operatorname{Im} \sum_{j=0}^{k-1}\left\langle N_{\epsilon}^{k-j-1}\left[H(t), N_{\epsilon}\right] N_{\epsilon}^{j} \varphi(t), N_{\epsilon}^{k} \varphi(t)\right\rangle
\end{aligned}
$$

where we have used

$$
\left[A, B^{2 k}\right]=\sum_{j=0}^{2 k-1} B^{j}[A, B] B^{2 k-j-1} .
$$

Since $Z^{j}(t)$ is bounded and $\left\|Z^{j}(\cdot)\right\| \in L_{l o c}^{1}(\mathbb{R})$ for $1 \leq j \leq k$, Lemma 2.2 (a) implies that $Z_{\epsilon}^{j}(t)$ is bounded for $1 \leq j \leq k$ and that $2 \sum_{j=1}^{k}\left\|Z_{\epsilon}^{j}(t)\right\| \leq$ const. $\sum_{j=1}^{k}\left\|Z^{j}(t)\right\| \equiv f_{k}(t)$, where $f_{k} \in L_{\text {loc }}^{1}(\mathbb{R})$ and is independent of $\epsilon$. It follows that

$$
\begin{aligned}
\left|(d / d t)\left\|N_{\epsilon}^{k} \varphi(t)\right\|^{2}\right| & \leq 2 \sum_{j=0}^{k-1}\left\|N_{\epsilon}^{k-j-1}\left[H(t), N_{\epsilon}\right] N_{\epsilon}^{j} \varphi(t)\right\|\left\|N_{\epsilon}^{k} \varphi(t)\right\| \\
& \leq 2 \sum_{j=0}^{k-1}\left\|Z_{\epsilon}^{k-j}(t)\right\|\left\|N_{\epsilon}^{k} \varphi(t)\right\|^{2} \\
& \leq f_{k}(t)\left\|N_{\epsilon}^{k} \varphi(t)\right\|^{2} .
\end{aligned}
$$

Integrating we obtain

$$
\left\|N_{\epsilon}^{k} \varphi(t)\right\| \leq\left\|N_{\epsilon}^{k} \varphi\right\| \exp \left|\frac{1}{2} \int_{s}^{t} f_{k}(u) d u\right| .
$$

We can now pass to the same argument as in the proof of Theorem 2.1 to conclude that $U(t, s)\left[\mathcal{D}\left(N^{k}\right)\right]=\mathcal{D}\left(N^{k}\right)$. 


\section{Applications.}

In this section we want to give some applications of the results of Section 2 to the Schrödinger equation

$$
i \frac{d}{d t} \varphi(t)=H(t) \varphi(t) ; \quad \varphi(s)=\varphi_{s}
$$

where $H(t)$ is the time-dependent Hamiltonian acting on the Hilbert space $\mathcal{H}=L^{2}\left(\mathbb{R}^{n}, d x\right)$.

We first consider Hamiltonians of the form

$$
H(t)=-\Delta+E(t) \cdot x+V(x, t) .
$$

We will restrict attention to electric fields $E(t): \mathbb{R} \rightarrow \mathbb{R}^{n}$ and potentials $V(x, t): \mathbb{R}^{n} \times \mathbb{R} \rightarrow \mathbb{R}$ obeying :
(i) $E(t)$ is differentiable.
(ii) $\left|\nabla_{x} V(x, t)\right| \leq f(t)(|x|+1)$ for some continuous function $f$.
(iii) the mapping $t \mapsto\left(x^{2}+1\right)^{-1} \frac{\partial V}{\partial t}(x, t) \in L^{\infty}\left(\mathbb{R}^{n}, d x\right)$ is continuous.

As for $N$, we take $N=p^{2}+x^{2}$, where $p=-i \nabla$. Note that the operator $N \geq 1$ and is self-adjoint on $\mathcal{D}(N)=\mathcal{D}\left(p^{2}\right) \cap \mathcal{D}\left(x^{2}\right)$. By Theorem 4 of Faris-Lavine [1], condition (ii) implies that $H(t)$ is essentially self-adjoint on $\mathcal{S}\left(\mathbb{R}^{n}\right)$, the space of $C^{\infty}$-functions on $\mathbb{R}^{n}$ rapidly decreasing at infinity, with domain $\mathcal{D}(H(t)) \supseteq \mathcal{D}(N)$. We remark that by the construction of the form domain, $\mathcal{Q}(N)=\mathcal{D}(|p|) \cap \mathcal{D}(|x|)$. Also, one can prove that $\mathcal{D}\left(N^{k}\right)=$ $\mathcal{D}\left(p^{2 k}\right) \cap \mathcal{D}\left(x^{2 k}\right)$ by integration by parts.

Given two Banach spaces $\mathcal{X}$ and $\mathcal{Y}$, we denote by $\mathcal{B}(\mathcal{X}, \mathcal{Y})$ the space of all bounded linear operators with domain $\mathcal{X}$ and range in $\mathcal{Y}$. For a multi-index $\alpha=\left(\alpha_{1}, \ldots, \alpha_{n}\right)$, where each $\alpha_{j}$ is a nonnegative integer, and $x=\left(x_{1}, \ldots, x_{n}\right) \in \mathbb{R}^{n}$, we put $|\alpha|=\alpha_{1}+\cdots+\alpha_{n}, x^{\alpha}=x_{1}^{\alpha_{1}} \cdots x_{n}^{\alpha_{n}}$ and $\nabla^{\alpha}=\left(\frac{\partial}{\partial x}\right)^{\alpha}=\left(\frac{\partial}{\partial x_{1}}\right)^{\alpha_{1}} \cdots\left(\frac{\partial}{\partial x_{n}}\right)^{\alpha_{n}}$. Let $B_{\infty}^{m}\left(\mathbb{R}^{n}\right)$ be the space of all $m$ times continuously differentiable functions $\varphi$ on $\mathbb{R}^{n}$ with bounded derivatives $\left(\frac{\partial}{\partial x}\right)^{\alpha} \varphi$ for $0<|\alpha| \leq m$. Our result is:

Theorem 3.1. Let $H(t)=-\Delta+E(t) \cdot x+V(x, t)$, where $E(t)$ and $V(x, t)$ obey conditions (i)-(iii) above, and let $N=p^{2}+x^{2}$. Then there exists a unique unitary propagator $U(t, s), t, s \in \mathbb{R}$, so that:

(a) for each $\varphi_{s} \in \mathcal{D}(N), \varphi(t)=U(t, s) \varphi_{s}$ is strongly differentiable and satisfies (3).

(b) $U(t, s)$ leaves $\mathcal{Q}(N)$ and $\mathcal{D}(N)$ invariant. 
If, in addition, $V(\cdot, t) \in B_{\infty}^{2 k}\left(\mathbb{R}^{n}\right)$ with $\left\|\left(\frac{\partial}{\partial x}\right)^{\alpha} V(x, \cdot)\right\|_{\infty} \in L_{\text {loc }}^{1}(\mathbb{R})$ for $0<|\alpha| \leq 2 k$, then $U(t, s)$ leaves $\mathcal{D}\left(N^{k}\right)$ invariant.

Proof. To prove the existence of the propagator, we define for $\varphi \in \mathcal{D} \equiv$ $\mathcal{D}(N),\|\varphi\|_{\mathcal{D}}=\|\varphi\|+\left\|p^{2} \varphi\right\|+\left\|x^{2} \varphi\right\|$. Then $\left(\mathcal{D},\|\cdot\|_{\mathcal{D}}\right)$ forms a Banach space which is continuously and densely embedded in $\mathcal{H}$. From (ii), we have $|V(x, t)| \leq \frac{1}{2} f(t) x^{2}+f(t)|x|+|V(0, t)|$. It follows by the continuity of $E, V$ and $f$ that on any compact interval $[-T, T]$, there are constants $a$ and $b$ so that $|E(t) \cdot x+V(x, t)| \leq a x^{2}+b$ for all $t \in[-T, T]$. Since

$$
\left\|p^{2} \varphi\right\|^{2}+\left\|c x^{2} \varphi\right\|^{2} \leq\left\|\left(p^{2}+c x^{2}\right) \varphi\right\|^{2}+2 c n\|\varphi\|^{2} \text { for } \varphi \in \mathcal{D}
$$

we see that if $c>a$, then $E(t) \cdot x+V(x, t)$ is $\left(p^{2}+c x^{2}\right)$-bounded with relative bound less than one. Thus, by the Kato-Rellich theorem, $H(t)+c x^{2}$ is self-adjoint on $\mathcal{D}$ for all $t \in[-T, T]$. Now, take $S(t)=H(t)+c x^{2}+i$. Then $S(t) \in \mathcal{B}(\mathcal{D}, \mathcal{H})$ is an isomorphism with $S(t) H(t) S(t)^{-1}=H(t)+G(t)$, where $G(t)=2 c i(p \cdot x+x \cdot p) S(t)^{-1} \in \mathcal{B}(\mathcal{H})$. By (i) and (iii), the mapping $t \mapsto S(t) \in \mathcal{B}(\mathcal{D}, \mathcal{H})$ is strongly differentiable. Also, a simple computation gives that

$$
\begin{aligned}
\| G(t)-G(u) H_{\mathcal{B}(\mathcal{H})} \leq & \|G(t)\|_{\mathcal{B}(\mathcal{H})}\|H(t)-H(u)\|_{\mathcal{B}(\mathcal{D}, \mathcal{H})}\left\|S(u)^{-1}\right\|_{\mathcal{B}(\mathcal{H}, \mathcal{D})} \\
\|H(t)-H(u)\|_{\mathcal{B}(\mathcal{D}, \mathcal{H})} \leq|E(t)-E(u)| & \quad+\left\|\left(x^{2}+1\right)^{-1}[V(x, t)-V(x, u)]\right\|_{L^{\infty}\left(\mathbb{R}^{n}, d x\right)} .
\end{aligned}
$$

Thus, by (i) and (iii), the mapping $t \mapsto H(t) \in \mathcal{B}(\mathcal{D}, \mathcal{H})$ and $t \mapsto G(t) \in$ $\mathcal{B}(\mathcal{H})$ are norm continuous. It follows from a classical result of Kato $([4]$, Theorem I) that there exists a unique unitary propagator $U(t, s)$ leaving $\mathcal{D}$ invariant so that (a) holds.

Next, we show that $U(t, s)$ leaves $\mathcal{Q}(N)$ invariant. We have seen that $\mathcal{D}(H(t)) \supseteq \mathcal{D}(N)$ for all $t$. So by Theorem 2.1, it suffices to show that $\pm i[H(t), N] \leq c(t) N$ for some locally integrable function $c(t)$. We compute $\pm i[H(t), N]$

$$
\begin{aligned}
& = \pm i\left\{\left[p^{2}, x^{2}\right]+\left[E(t) \cdot x, p^{2}\right]+\left[V(x, t), p^{2}\right]\right\} \\
& = \pm\left\{2(p \cdot x+x \cdot p)-2 E(t) \cdot p-\left(p \cdot \nabla_{x} V(x, t)+\nabla_{x} V(x, t) \cdot p\right)\right\} \\
& \leq 2\left(p^{2}+x^{2}\right)+p^{2}+|E(t)|^{2}+p^{2}+\left|\nabla_{x} V(x, t)\right|^{2} \\
& \leq\left\{4+|E(t)|^{2}+4 f(t)^{2}\right\} N
\end{aligned}
$$

as required, where we have used condition (ii) and the fact that $N \geq 1$.

Finally, we prove the last statement of the theorem. Let

$$
\Gamma \equiv L_{l o c}^{1}(\mathbb{R}, d t ; \mathcal{B}(\mathcal{H}))
$$


By Theorem 2.3, it suffices to show that if $V(\cdot, t) \in B_{\infty}^{2 k}\left(\mathbb{R}^{n}\right)$ with $\left\|\left(\frac{\partial}{\partial x}\right)^{\alpha} V(x, \cdot)\right\|_{\infty} \in L_{l o c}^{1}(\mathbb{R})$ for $0<|\alpha| \leq 2 k$, then

$$
Z^{j}=N^{j-1}[H(\cdot), N] N^{-j} \in \Gamma
$$

for $1 \leq j \leq k$. We prove this inductively. Let $D=p \cdot x+x \cdot p$ be the dilation operator. Since

$$
\begin{aligned}
Z^{1}(t) & =[H(t), N] N^{-1} \\
& =-2 i\left\{D-E(t) \cdot p-\nabla_{x} V(x, t) \cdot p+\frac{i}{2} \Delta_{x} V(x, t)\right\} N^{-1},
\end{aligned}
$$

the case $k=1$ follows easily from the closed graph theorem and the hypotheses on $E$ and $V$. Now consider the case of general $k \geq 2$. By the induction hypothesis, we have $Z^{j} \in \Gamma$ for $1 \leq j \leq k-1$. So, we need only prove that $Z^{k} \in \Gamma$. By Lemma $2.2(\mathrm{~b})$, it is sufficient to prove that $\left\{(\operatorname{ad} N)^{k} H(\cdot)\right\} N^{-k} \in \Gamma$. We compute on $\mathcal{S}\left(\mathbb{R}^{n}\right)$ :

$$
(\operatorname{ad} N)^{2} H(t)=4\left\{\begin{array}{c}
2\left(p^{2}-x^{2}\right)+E(t) \cdot x+\nabla_{x} V(x, t) \cdot x+\frac{1}{4} \Delta_{x}^{2} V(x, t) \\
-\sum_{j=1}^{n}\left(\nabla_{x} \frac{\partial V}{\partial x_{j}}(x, t)\right) \cdot p p_{j}+i \nabla_{x}\left(\Delta_{x} V(x, t)\right) \cdot p
\end{array}\right\}
$$

where we have used the following basic identities:

$$
\begin{gathered}
{[N, D]=4 i\left(x^{2}-p^{2}\right),[N, E(t) \cdot p]=2 i E(t) \cdot x,[N, E(t) \cdot x]=-2 i E(t) \cdot p} \\
{\left[p^{2}, W(x)\right]=-2 i \nabla W \cdot p-\Delta W,\left[x^{2}, \nabla W(x) \cdot p\right]=2 i \nabla W \cdot x} \\
{\left[p^{2}, \nabla W(x) \cdot p\right]=-2 i \sum_{j=1}^{n}\left(\nabla \frac{\partial W}{\partial x_{j}}\right) \cdot p p_{j}-\nabla(\Delta W) \cdot p .}
\end{gathered}
$$

By repeated application of these formulas, we find that $(\operatorname{ad} N)^{k} H(t)$ is a linear combination of operators of the form:

$$
p^{2}-x^{2}(\text { or } D), E(t) \cdot x(\text { or } E(t) \cdot p) \text { and }\left[\left(\frac{\partial}{\partial x}\right)^{\alpha} V(x, t)\right] x^{\beta} p^{\gamma}
$$

where $0<|\alpha| \leq 2 k,|\beta| \leq k / 2$ and $|\gamma| \leq k$. Since $x^{\beta} p^{\gamma} N^{-k}$ is bounded on $\mathcal{H}$ so long as $|\beta| \leq k$ and $|\gamma| \leq k$, the hypotheses of $E$ and $V$ now imply that $\left\{(\operatorname{ad} N)^{k} H(\cdot)\right\} N^{-k} \in \Gamma$. This completes the proof.

Corollary 3.2. In Theorem 3.1, if $V(\cdot, t)$ is a $C^{\infty}$-function on $\mathbb{R}^{n}$ with bounded derivatives and $\left\|\left(\frac{\partial}{\partial x}\right)^{\alpha} V(x, \cdot)\right\|_{\infty} \in L_{\text {loc }}^{1}(\mathbb{R})$ for all $\alpha \neq 0$, then $U(t, s)$ leaves $\mathcal{S}\left(\mathbb{R}^{n}\right)$ invariant.

Proof. The corollary follows immediately from the fact that

$$
\mathcal{S}\left(\mathbb{R}^{n}\right)=\cap_{k=1}^{\infty} \mathcal{D}\left(N^{k}\right)
$$


In the remainder of this section, we want to give an application to Hamiltonians of the form

$$
H(t)=-\Delta+V(x, t) .
$$

We will assume potentials $V(x, t): \mathbb{R}^{n} \times \mathbb{R} \rightarrow \mathbb{R}$ obeying:

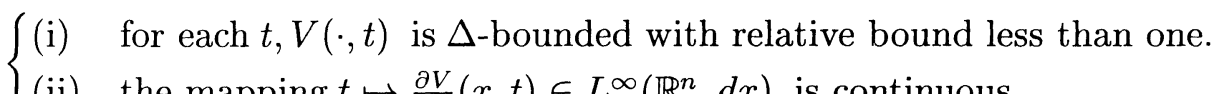

(ii) the mapping $t \mapsto \frac{\partial V}{\partial t}(x, t) \in L^{\infty}\left(\mathbb{R}^{n}, d x\right)$ is continuous.

Notice that condition (i) and the Kato-Rellich theorem imply that $H(t)$ is essentially self-adjoint on $\mathcal{S}\left(\mathbb{R}^{n}\right)$ with domain $\mathcal{D}(H(t))=\mathcal{D}(\Delta)$. Corresponding to Theorem 3.1, we have:

Theorem 3.3. Let $H(t)=-\Delta+V(x, t)$, where $V(x, t)$ obeys conditions (i) and (ii) above. Then there is a unique unitary propagator $U(t, s), t, s \in \mathbb{R}$, leaving $\mathcal{D}(\Delta)$ invariant so that for each $\varphi_{s} \in \mathcal{D}(\Delta), \varphi(t)=U(t, s) \varphi_{s}$ is strongly differentiable and satisfies (3). Moreover,

(a) If $\left|\nabla_{x} V(x, t)\right| \leq f(t)$ for some continuous $f$, then $U(t, s)$ leaves $\mathcal{Q}(-\Delta)$ invariant.

(b) If $V(\cdot, t) \in B_{\infty}^{2 k}\left(\mathbb{R}^{n}\right)$ with $\left\|\left(\frac{\partial}{\partial x}\right)^{\alpha} V(x, \cdot)\right\|_{\infty} \in L_{\text {loc }}^{1}(\mathbb{R})$ for $0<|\alpha| \leq 2 k$, then $U(t, s)$ leaves $\mathcal{D}\left(\Delta^{k}\right)$ invariant.

Proof. The proof of the existence statement closely parallels the proof given in Theorem 3.1 except that we choose $\mathcal{D}=\mathcal{D}(\Delta), \quad S(t)=H(t)+i$ and define $\|\varphi\|_{\mathcal{D}}=\|\varphi\|+\left\|p^{2} \varphi\right\|$ so that $S(t) H(t) S(t)^{-1}=H(t)$. Then one proves that the mapping $t \mapsto S(t) \in \mathcal{B}(\mathcal{D}, \mathcal{H})$ is strongly differentiable and that the mapping $t \mapsto H(t) \in \mathcal{B}(\mathcal{D}, \mathcal{H})$ is norm continuous as before. To prove (a) and (b), we take $N=-\Delta+1$. In case (a), since

$$
\begin{aligned}
\pm i[H(t), N] & =\mp\left\{p \cdot \nabla_{x} V(x, t)+\nabla_{x} V(x, t) \cdot p\right\} \\
& \leq p^{2}+\left|\nabla_{x} V(x, t)\right|^{2} \leq\left\{1+f(t)^{2}\right\} N,
\end{aligned}
$$

Theorem 2.1 implies that $U(t, s)$ leaves $\mathcal{Q}(N)=\mathcal{Q}(-\Delta)$ invariant. In case (b), the computations similar to those used in Theorem 3.1 show that $(\operatorname{ad} N)^{k} H(t)$ is a linear combination of operators of the form: $\left[\left(\frac{\partial}{\partial x}\right)^{\alpha} V(x, t)\right] p^{\gamma}$, where $0<|\alpha| \leq 2 k$ and $|\gamma| \leq k$. Thus by hypothesis, we have

$$
\left\{(\operatorname{ad} N)^{k} H(\cdot)\right\} N^{-k} \in L_{l o c}^{1}(\mathbb{R}, d t ; \mathcal{B}(\mathcal{H})) .
$$

Again, following the proof of Theorem 3.1, we conclude that $U(t, s)$ leaves $\mathcal{D}\left(N^{k}\right)=\mathcal{D}\left(\Delta^{k}\right)$ invariant. 
Acknowledgments. The author wishes to thank the referee for useful comments.

\section{References}

[1] W.G. Faris and R.B. Lavine, Commutators and self-adjointness of Hamiltonian operators, Comm. Math. Phys., 35 (1974), 39-48.

[2] J. Fröhlich, Application of commutator theorems to the integration of representations of Lie algebras and commutation relations, Comm. Math. Phys., 54 (1977), 135150.

[3] W. Hunziker, On the space-time behavior of Schrödinger wavefunctions, J. Math. Phys., 7 (1966), 300-304.

[4] T. Kato, Linear evolution equations of hyperbolic type I,II, J. Fac. Sci. Univ. of Tokyo, Sec. IA, 17 (1970), 241-258; J. Math. Soc. Japan, 25 (1973), 648-666.

[5] S.T. Kuroda and H. Morita, An estimate for solutions of Schrödinger equations with time-dependent potentials and associated scattering theory, J. Fac. Sci. Univ. of Tokyo, Sec. IA, 24 (1977), 459-475.

[6] T. Ozawa, Invariant subspaces for the Schrödinger evolution group, Ann. Inst. Henri Poincaré, Sec. A, 54 (1991), 43-57.

[7] Space-time behavior of propagators for Schrödinger evolution equations with Stark effect, J. Funct. Anal., 97 (1991), 264-292.

[8] C. Radin and B. Simon, Invariant domains for the time-dependent Schrödinger equation, J. Diff. Eq., 29 (1978), 289-296.

[9] M. Reed and B. Simon, Methods of Modern Mathematical Physics II, Fourier Analysis, Self-Adjointness, Academic Press, New York, 1975.

[10] C.H. Wilcox, Uniform asymptotic estimates for wave packets in the quantum theory of scattering, J. Math. Phys., 6 (1965), 611-620.

[11] K. Yajima, Existence of solutions for Schrödinger evolution equations, Comm. Math. Phys., 110 (1987), 415-426.

Received December 20, 1993 and revised April 19, 1994.

National Tsing Hua University

HSINCHU, TAIWAN 30043

REPUBLIC OF China 



\title{
PACIFIC JOURNAL OF MATHEMATICS
}

\author{
Founded in 1951 by
}

\author{
E. F. Beckenbach (1906-1982) $\quad$ F. Wolf (1904-1989)
}

\section{EDITORS}

Sun-Yung A. Chang (Managing Editor) Robert Finn University of California

Los Angeles, CA 90095-1555

pacific@math.ucla.edu

\section{F. Michael Christ}

University of California

Los Angeles, CA 90095-1555

christ@math.ucla.edu

Nicholas Ercolani

University of Arizona

Tucson, AZ 85721

ercolani@math.arizona.edu
Stanford University

Stanford, CA 94305

finn@gauss.stanford.edu

Steven Kerckhoff

Stanford University

Stanford, CA 94305

spk@gauss.stanford.edu

Martin Scharlemann

University of California

Santa Barbara, CA 93106

mgscharl@math.ucsb.edu

\section{Gang Tian}

Massachusettes Institute of Technology

Cambridge, MA 02139

tian@math.mit.edu

\section{V.S. Varadarajan}

University of California

Los Angeles, CA 90095-1555

vsv@math.ucla.edu

Dan Voiculescu

University of California

Berkeley, CA 94720

dvv@math.berkeley.edu

\section{SUPPORTING INSTITUTIONS}

CALIF. INST. OF TECHNOLOGY CHINESE UNIV. OF HONG KONG MACQUARIE UNIVERSITY NEW MEXICO STATE UNIV. OREGON STATE UNIV. PEKING UNIVERSITY RITSUMEIKAN UNIVERSITY STANFORD UNIVERSITY TOKYO INSTITUTE OF TECHNOLOGY UNIVERSIDAD DE LOS ANDES
UNIV. OF ARIZONA

UNIV. OF BRITISH COLUMBIA

UNIV. OF CALIF., BERKELEY

UNIV. OF CALIF., DAVIS

UNIV. OF CALIF,, IRVINE UNIV. OF CALIF., LOS ANGELES

UNIV. OF CALIF., RIVERSIDE UNIV. OF CALIF., SAN DIEGO UNIV. OF CALIF., SANTA BARBARA UNIV. OF CALIF., SANTA CRUZ
UNIV. OF HAWAII

UNIV. OF MELBOURNE

UNIV. OF MONTANA

UNIV. NACIONAL AUTONOMA DE MEXICO

UNIV. OF NEVADA, RENO

UNIV. OF OREGON

UNIV. OF SOUTHERN CALIFORNIA

UNIV OF UTAH

UNIV. OF WASHINGTON

WASHINGTON STATE UNIVERSITY

The supporting Institutions listed above contribute to the cost of publication of this Journal, but they are not owners or publishers and have no responsibility for its contents or policies.

Manuscripts must be prepared in accordance with the instructions provided on the inside back cover.

The table of contents and the abstracts of the papers in the current issue, as well as other information about the Pacific Journal of Mathematics, may be found on the Internet at http://www.math.uci.edu/pjm.html.

The Pacific Journal of Mathematics (ISSN 0030-8730) is published monthly except for July and August. Regular subscription rate: $\$ 245.00$ a year (10 issues). Special rate: $\$ 123.00$ a year to individual members of supporting institutions.

Subscriptions, back issues published within the last three years and changes of subscribers address should be sent to Pacific Journal of Mathematics, P.O. Box 4163, Berkeley, CA 94704-0163, U.S.A. Prior back issues are obtainable from Kraus Periodicals Co., Route 100, Millwood, NY 10546.

The Pacific Journal of Mathematics at the University of California, c/o Department of Mathematics, 981 Evans Hall, Berkeley, CA 94720 (ISSN 0030-8730) is published monthly except for July and August. Second-class postage paid at Berkeley, CA 94704, and additional mailing offices. POSTMASTER: send address changes to Pacific Journal of Mathematics, P.O. Box 6143, Berkeley, CA 94704-0163.

PUBLISHED BY PACIFIC JOURNAL OF MATHEMATICS at University of California, Berkeley, CA 94720, A NON-PROFIT CORPORATION

This publication was typeset using AMS-LATEX,

the American Mathematical Society's TEX macro system.

Copyright (c) 1995 by Pacific Journal of Mathematics 


\title{
PACIFIC JOURNAL OF MATHEMATICS
}

\author{
Volume $175 \quad$ No. $1 \quad$ September 1996
}

Homogeneous Ricci positive 5-manifolds

Dimitri Alekseevsky, Isabel Dotti de Miatello and CARlos J.

FERRARIS

On the structure of tensor products of $\ell_{p}$-spaces

ALVARO ARIAS and JEFFREY D. FARMER

The closed geodesic problem for compact Riemannian 2-orbifolds

JosEPH E. BORZELLINO and BENJAMIN G. LORICA

Small eigenvalue variation and real rank zero

Ola Bratteli and George A. Elliott

Global analytic hypoellipticity of $\square_{b}$ on circular domains

So-CHIN CHEN

Sharing values and a problem due to C. C. Yang

XIN-Hou HuA

Commutators and invariant domains for Schrödinger propagators

Min-JEI HUANG

Chaos of continuum-wise expansive homeomorphisms and dynamical properties of sensitive maps of graphs

\section{HiS AO KATO}

Some properties of Fano manifolds that are zeros of sections in homogeneous vector bundles over Grassmannians

\section{OLIVER KÜCHLE}

On polynomials orthogonal with respect to Sobolev inner product on the unit circle

XIN Li and FRANCISCO MARCELLAN

Maximal subfields of $\mathbf{Q}(i)$-division rings

STEVEN LIEDAHL

Virtual diagonals and $n$-amenability for Banach algebras

AlAN L. T. PATERSON

Rational Pontryagin classes, local representations, and $K^{G}$-theory

Claude Schochet

An equivalence relation for codimension one foliations of 3-manifolds

SANDRA SHIELDS

A construction of Lomonosov functions and applications to the invariant subspace problem

\section{ALEKSANDER SIMONIČ}

Complete intersection subvarieties of general hypersurfaces 